\title{
Radiation Oncology reviewer acknowledgement 2015
}

Claus Belka ${ }^{1}$ and Sarah M. Theissen ${ }^{2^{*}}$

\section{Contributing reviewers}

The editorial team of Radiation Oncology would like to thank all our reviewers who have contributed to the journal in 2015. Without the participation of skilful reviewers, no academic journal could succeed, and we are grateful to the committed individuals who have given their time and expertise to the peer review of manuscripts for Radiation Oncology. We look forward to your continued support in 2016.

\author{
J Abayomi \\ UK
}

Takanori Abe

Japan

Aruna Abhyankar

UK

Yasser Abo-Madyan

Germany

Sonja Adebahr

Germany

Sebastian Adeberg

Germany

Vikram Adhikarla

USA

Sushma Agrawal

India

Markus Alber

Denmark

BM Aleman

Netherlands

Michelle Alonso-Basanta

USA

\author{
Shafak Aluwini \\ Netherlands
}

\section{S Alvi}

UK

Indu Ambudkar

USA

M Amsbaugh

USA

Nicolaus Andratschke

Germany

Stefano Arcangeli

Italy

Moran Artzi
Israel

Michael Atkinson

Germany

Marianne Aznar

Denmark

\section{Houda Bahig}

Canada

Kurt Baier

Germany

\section{Elizabeth Baldini \\ USA}

Hendrik Ballhausen

Germany

\section{Mark Bangert}

Germany

Carlos Barcenas
USA

Tomasz Barelkowski

Germany

M Baron Nelson

USA

Guido Baroni

Italy

Glenn Bauman

Canada

Wolfgang Baus

Germany

\section{P Bayo}

Germany

Jose Bazan

USA

\footnotetext{
* Correspondence: sarah.theissen@biomedcentral.com

'Department of Radiation Oncology, University Hospital of Munich,

Marchioninistr. 15, 81377 Munich, Germany

2BioMed Central, 236 Gray's Inn Road, London WC1X 8HB, UK
} 
Marcus Beck

Germany

Laurent Bedenne

USA

GL Beets

Netherlands

Jose Belderbos

Netherlands

ML Belli

Italy

J Berenson

USA

Jannette Beukema

Netherlands

Wolfgang Birkfellner

Austria

Marc Bischof

Germany

Pierre Blanchard

France

Oliver Blanck

Germany

Lennart Blomqvist

Sweden

Judit Boda-Heggemann

Germany

Raphael Bodensohn

USA

Beat Bojaxhiu

Switzerland

William Bonner

USA

JP Boström

Germany

Herbert Braselmann

Germany

$S$ Breedveld

Netherlands

Michael Bremer

Germany

CL Brouwer

Netherlands

Michelle Brown

Switzerland
T Brown

Australia

Stephen Brown

USA

Thomas Brunner

Germany

J Buatti

USA

Grete Buchholz

Germany

Wilfried Budach

Germany

Michela Buglione

Italy

Irene A. Burger

Switzerland

Karl Butterworth

UK

Florian Büttner

Germany

Aras Emre Canda

Turkey

W Cao

USA

Joel Castelli

France

C Castoro

Italy

Jimmy Caudell

USA

Jean-Michel Caudrelier

Canada

Laura Cella

Italy

Giovanna Cenacchi

Italy

Laura Cervino

USA

S Chakraborty

India

Mark Ka Heng Chan

Hong Kong

Samuel Chao

USA
Naved Chaudhri

Germany

L Chien

Taiwan

Hyeon-Mih Cho

South Korea

Youngmin Choi

South Korea

Christos Chouaid

France

James Chow

Canada

F Cicone

Italy

Ilja Ciernik

Germany

Nikola Cihoric

Switzerland

Mario Ciocca

Italy

S Clemente

Italy

Brian Collins

USA

Sean Collins

USA

Anna Constantinescu

Germany

M Coskun-Breuneval

Turkey

Felipe Couñago

Spain

Luca Cozzi

Italy

Gilles Crehange

France

Richard Crevenna

Austria

Johannes Crezee

Netherlands

SB Crowe

Australia

Alessandra Curioni

Switzerland 
Jakub Cvek

Czech Republic

Clemens Cyran

Germany

Brian Czito

USA

Giuseppe D'agostino

Italy

Alan Dal Pra

Switzerland

Sidsel Damkjaer

Denmark

Rolando M. D'Angelillo

Italy

Cyril Danjoux

Canada

S Dashti

USA

Niloyranjan Datta

Switzerland

$P$ De Ieso

Australia

Reinhard Depping

Germany

Luc Dewit

Netherlands

K Dholam

India

Jennifer Dhont

Belgium

Alexander Dietrich

Germany

Sarah Differding

Belgium

Andreas Dinkel

Germany

Tcholpon Djuzenova

Germany

Sophie Dobiasch

Germany

Barbara Dobler

Germany

H Doi

Japan
Olivio Donati

Switzerland

A Donneys

USA

Wolfgang Dörr

Germany

Laura Doyle

USA

Guido Drexler

Germany

Laurence Dubrez

France

Yvonne Dzierma

Germany

F Ebner

Germany

Ardalan Ebrahimi

Australia

K Eda

Turkey

A Eisbruch

USA

Harriet Eldredge-Hindy

USA

John Eley

USA

Mostafa Elhaddad

Egypt

Olgun Elicin

Switzerland

Peter Ellis

Canada

Andrew Elson

USA

Wolfgang Enghardt

Germany

J Eriksen

Denmark

Iris Ernst

Germany

Birgit Ertl-Wagner

Germany

Marion Essers

Netherlands
Laura Evangelista

Italy

Khashayar Fakhrian

Germany

K Farr

Denmark

G Fastner

Austria

Dimitrios Fatouros

Greece

Giovanni Fattori

Switzerland

J Feddock

USA

Roman Fehr

Germany

Pascal Fenoglietto

France

MA Fernández-Seara

Spain

Petra Feyer

Germany

Jacob Finkelstein

USA

Alba Fiorentino

Italy

Michael Fix

Switzerland

Daniel Fleischmann

Germany

Ralf Floca

Germany

Tullio Florio

Italy

Robert Foerster

Germany

Antonella Fogliata

Italy

Emmanouil Fokas

UK

Davide Fontanarosa

Netherlands

M Foote

Australia 
P Franco

Italy

Nicolaas Franken

Netherlands

Thorsten Frenzel

Germany

Benjamin Frey

Germany

Anna Friedl

Germany

Thomas Friedrich

Germany

Weihua Fu

USA

M Fujiwara

Japan

M Fukumoto

Japan

Simone Fulda

Germany

Guenther Gademann

Germany

Udo Gaipl

Germany

Razvan Galalae

Germany

Maria Antonietta Gambacorta

Italy

Gregory Gan

USA

Ute Ganswindt

Germany

Kevin Gardner

USA

Elisabetta Gargioni

Germany

Cristina Garibaldi

Italy

Tobias Gauer

Germany

Cengiz Gemici

Turkey

Michael Gensheimer

USA
Dietmar Georg

Austria

PC Gerszten

USA

Pirus Ghadjar

Germany

Sarbani Ghosh Laskar

India

David Gierga

USA

Suki Gill

Australia

Frank Giordano

Germany

Meredith Giuliani

Canada

Christoph Glanzmann

Switzerland

Daniel Gomez

USA

J Gómez-Millán

Spain

Holger Gottschlag

Germany

Gerhard Grabenbauer

Germany

Christian Graeff

Germany

Clemens Grassberger

USA

Peter Greer

Australia

Arne Gruen

Germany

M Gryzinski

Poland

G Guidi

Italy

JL Guinot

Spain

Marianne Guren

Norway

Cristina Gutierrez

Spain
Antonio Gutierrez

Spain

Christian Gutschow

Switzerland

N Gyldenkerne

Denmark

Rick Haas

Netherlands

Daniel Habermehl

Germany

Gregor Habl

Germany

Fred Hacker

USA

Marlen Haderlein

Germany

Bruce Haffy

USA

Matthias Häfner

Germany

Ester Hammond

UK

Jean-Michel Hannoun-Levi

France

Matthew Harkenrider

USA

Matthias Hartmann

Switzerland

Takayuki Hashimoto

Japan

Henrik Hauswald

Germany

Maria Hawkins

UK

S He

China

Rolf A. Heckemann

UK

Christine Heinrich

Germany

Miachael Henke

Germany

Willers Henning

USA 
Klaus Herfarth

Germany

Julia Heß

Germany

Shuichi Hironaka
Japan

Ying Hitchcock

USA

Holger Hof

Germany

Jan Hofmaier

USA

W Hofstetter

USA

Yoshitaka Honma

Japan

S Hoogcarspel

Netherlands

Mohammad B Hossain

USA

Stephan Huber

Germany

Florence Huguet

France

David Hui

USA

Michael Ingrisch

Germany

Peter Inskip

USA

Edy Ippolito

Italy

Satoshi Ishikura

Japan

Hiromichi Ishiyama

Japan

Fumiaki Isohashi

Japan

Rolf D. Issels

Germany

Julian Jacob

France

Aaron Jacobs

USA
Stefan Janssen

Switzerland

Verena Jendrossek

Germany

Alexandra Jensen

Switzerland

Xun Jia

USA

Hosang Jin

USA

Keiichi Jingu

Japan

Lang Jingyi

China

Mirjana Josipovic

Denmark

An Jusheng

China

Johannes Kaanders

Netherlands

Ulf Kahlert

Germany

A Kakria

India

Sanjeeva Kalva

USA

MK Kam

USA

Florian Kamp

Germany

Severin Kampfer

Germany

Josephine Kang

USA

Steffi Kantz

Germany

Tom Karagiannis

Australia

I Karam

Canada

Sonja Katayama

Germany

David Kaul

Germany
Chul-Seung Kay

South Korea

T Kehwar

USA

Monika Keller

Germany

M Kent

USA

Lucyna Kepka

Poland

Oliver Kepp

France

Sarah Kerns

USA

Ki Chang Keum

South Korea

R Keus

Netherlands

E Khalil

Egypt

M Khan

USA

Nikolai Khodarev

USA

Divya Khosla

India

Philipp Kickingereder USA

YH Kim

North Korea

NamKyu Kim

South Korea

Yong Bae Kim

South Korea

Grace Kim

USA

Tari King

USA

Rainer Klement

Germany

Stephan Kloeck

Switzerland

Sebastian Klüter

Germany 
Shirley Knauer

Germany

Stefan Knippen

Germany

Thomas Koch

Germany

Martin Kocher

Germany

Fumitaka Koga

Japan

David Koh

USA

H Kok

Netherlands

Vassilis Kouloulias

Greece

Mechthild Krause

Germany

Robert Krempien

Germany

Marco Krengli

Italy

Jan Kriz

Germany

David Krug

Germany

Kevin Krull

USA

Marko Krznaric

UK

Sebastian Kuger

Germany

A Kunwar

Canada

Joseph Kurian

USA

George Kyrgias

Greece

Guillaume Landry

Germany

Stephanie Lang

Switzerland

J van Lanschot

Netherlands
Jung-Il Lee

South Korea

Ik Jae Lee

South Korea

Andrew Lee

USA

$P$ Leone

USA

Vera Levina

USA

Antonin Levy

France

John Lewis

USA

Baosheng Li

China

Minglun Li

Germany

Jianbin Li

China

Karen Liby

USA

Do Hoon Lim

South Korea

M Lim

South Korea

$S$ Lin

USA

Jin-Ching Lin

Taiwan

Katja Lindel

Germany

Claudia Linsenmeier

Switzerland

Jun Liu

China

Chao Liu

USA

Kristina Loessl

Switzerland

Frank Lohr

Germany

A Lollert

Germany
M Lopes

Brazil

Jose López Torrecilla

Spain

Alexander Louie

Canada

Dorota Lubgan

USA

Johannes Lutterbach

Germany

Stephen Lutz

USA

J Ma

China

Andreas Mack

Switzerland

K Maeda

Japan

Angelo Maggio

Italy

Umesh Mahanshetty

India

Cornelius Maihöfer

Germany

Philippe Maingon

France

Raymond Mak

USA

Venkata Manem

Canada

M Maraldo

Denmark

Boris Margulis

Russia

Maurie Markman

USA

Simone Marnitz

Germany

J Marsh

USA

David Mathieu

Canada

Christiane Matuschek

Germany 
M McDonald

USA

Andrew McDonald

USA

Icro Meattini

Italy

Alejandra Mendez Romero

Netherlands

Valeria Mercadante

UK

P Metcalfe

Australia

Bernhard Meyer

Germany

J Michel

France

Oliver Micke

Germany

Ben Mijnheer

Netherlands

Michael Milano

USA

Giuseppe Minniti

Italy

Raymond Miralbell

Switzerland

Rene-Olivier Mirimanoff

Switzerland

Martin Misslbeck

Germany

Yasuyoshi Miyata

Japan

Anouchka Modesto

France

Raphaël Moeckli

Switzerland

Simone Moertl

Germany

Tan-Lucien Mohammed

USA

Majid Mohiuddin

USA

Konrad Mohnike

Germany
Michael Molls

Germany

F Moretto

Italy

Alessio Giuseppe Morganti

USA

Lutz Moser

Germany

Thorsten Moser

Germany

Filipe Moura

Portugal

Michael Moyers

USA

Petra Mozes

Germany

Nikola Mueller

Germany

Arndt-Christian Müller

Germany

Klaus Müller

Germany

Reinhold Müller

Germany

Olaf Nairz

Austria

N Nakajima

Japan

Pierina Navarria

Italy

Dunlap Neal

USA

VP Nelamangala Ramakrishnaiah India

Nicole Nesvacil

Austria

Nam Nguyen

USA

R. Charles Nichols

USA

Geoffrey Nichols

USA

Giorgia Nicolini

USA
Carsten Nieder

Norway

Gabriele Niedermann

Germany

Peter Niehoff

Germany

Reinoud Nijhuis

Germany

A Nikoghosyan

Germany

K Nikolajek

Germany

Maximilian Niyazi

Germany

S Nougaret

France

Tuathan O'Shea

UK

Markus Oechsner

Germany

Christian Okoye

USA

Robert Olson

Canada

Ester Orlandi

Italy

Michael Orth

Germany

Feras Oskan

Germany

Piet Ost

Belgium

O Oyeyemi

Nigeria

Y Ozawa

Japan

Mahmut Ozsahin

Switzerland

Timothy Padera

USA

Jianji Pan

China

Herb Pang

Hong Kong 
Mike Partridge

UK

David Pasquier

France

Margarethus Paulides

Netherlands

Arnold Paulino

USA

Frank Paulsen

Germany

Ruth Paulssen

Norway

A Paumier

France

D Peiffert

USA

Vincent Pennaneach

France

Evangelia Peponi

Greece

Francesco Perri

Italy

Peter Petersen

Denmark

Heike Peulen

Netherlands

Asja Pfaffenberger

Germany

C Philippson

Belgium

B Pieters

Netherlands

Michael Pinkawa

Germany

M Pinkham

Australia

Tomasz Piotrowski

Poland

Ludwig Plasswilm

Switzerland

George Plataniotis

UK

Volker Platz

Germany
Buelent Polat

Germany

Csaba Polgar

Hungary

A Pontoriero

Italy

Ramachandran Prabhakar

India

Matthias Prall

Germany

Heru Prasetio

Germany

Jochen Prehn

Ireland

Brendan Prendergast

USA

Vesna Prokic

Germany

Martin Pruschy

Switzerland

Pedro Puig

Spain

Paul Martin Putora

Switzerland

Florian Putz

Germany

Sharon Qi

USA

Cornelis Raaijmakers

Netherlands

Ulla Ramm

Germany

Andrew Rankin

USA

Coen Rasch

Netherlands

Paul Read

USA

Peter Reher

Australia

Michael Reiner

Germany

Marco Riboldi

Italy
Patrick Richard

USA

Andreas Richter

Germany

Daniel Richter

Germany

Juliane Rieber

Germany

Harald Rief

Germany

Stefan Rieken

Germany

Oliver Riesterer

Switzerland

Andreas Rimner

USA

Mark Rivard

USA

Mack Roach

USA

David Roberge

Canada

Stephen Roberts

UK

Nathalie Rochet

Germany

Franz Rödel

Germany

Dirk Roggenbuck

Germany

Jesse Roman

USA

Nicola Rosenfelder

UK

Stephan Roth

Germany

Kai Rothkamm

Germany

Carmen Rubio

Spain

Justine Rudner Germany

Tatjana Rundek

USA 
Elvio Grazioso Russi
Italy

Sebastia Sabater

Spain

Mohammadsaeed Saboori

Germany

Arjun Sahgal

Canada

Joseph Salama

USA

Ladan Saleh-Ebrahimi

Germany

Henning Salz

Germany

D Sapkaroski

Australia

Ryohei Sasaki

Japan

Dörthe Schaue

USA

Heike Scheithauer

Germany

Devin Schellenberg

Canada

Michel Schlienger

France

Thomas Schmid

Germany

Heinz Schmidberger

Germany

M Schmidt

USA

Uwe Schneider

Switzerland

Michael Scholz

Germany

Ed Schwalbe

UK

V Scotti

Italy

Felix Sedlmayer

Austria

Petra Seibold

Germany
Max Seidensticker

Germany

A Sekine

Japan

Robert Semrau

Germany

Sabine Semrau

Germany

Suresh Senan

Netherlands

Matteo Seregni

Italy

A Serna

Spain

Marco Serpa

Germany

D Severin

Canada

Daya Nand Sharma

India

Gregory Sharp

USA

J Sheehan

USA

David Shepard

USA

Helen Shih

USA

T Showalter

USA

Pei-Wei Shueng

Taiwan

David Shultz

USA

Frank-Andre Siebert

Germany

Anurag Singh

USA

Ira-Ida Skvortsova

Austria

Nicholas Slevin

UK

Robert Smee

Australia
Wendy Smith

Canada

Matthias Soehn

Germany

Dennis Sohn

Germany

Y Song

China

Jan-Jakob Sonke

Netherlands

Yu Yang Soon

Singapore

Stefan Speer

Germany

Michael Spiotto

USA

P Srivastava

India

Kevin Stephans

USA

Florian Sterzing

Germany

Florian Stieler

Germany

Alison Stillie

UK

Sandro Stoeckli

Switzerland

Guy Storme

Belgium

Christoph Straube

Germany

Tino Streller

Switzerland

Vratislav Strnad

Germany

Carmen Stromberger

Germany

Gabriela Studer

Switzerland

Martin Stuschke

Germany

Kristin Stützer

Germany 
Isolde Summerer

Germany

K Sun

China

K Suzuki

Japan

O Suzuki

Japan

SG Swarts

USA

Marta Switlyk

Norway

Ghazaleh Tabatabai

Germany

P Tai

Canada

Oike Takahiro

Japan

M Takeuchi

Japan

Yamei Tang

China

Mohmad Ashraf Teli

India

Loukas Thanos

Greece

Gudrun Theile

Switzerland

Christian Thieke

Germany

Hubert Thierens

Belgium

Christopher Thomas

UK

J Thompson

Australia

Zhen Tian

USA

Carmen Timke

Germany

Ingeborg Tinhofer

Germany

Manuel Todorovic

Germany
J Torok

USA

Almut Troeller

Germany

Klaus Trott

Germany

MH Truong

USA

Nikolaos Tselis

Germany

Alexandros Tsikkinis

Switzerland

Mitsutoshi Tsukimoto

Japan

Deniz Tural

Turkey

L Turcotte

USA

B Turkbey

USA

Mario Turri-Zanoni

Italy

Kristian Unger

Germany

Laura Van den Bergh

Belgium

Jacoba van der Zee

Netherlands

S van Dyk

Australia

L van Roozendaal

Netherlands

Jef Vandemeulebroucke

Belgium

$\mathrm{N}$ vanderWalde

USA

V Vassiliou

Greece

Peter Vaupel

Germany

Jirina Vavrova

Czech Republic

Wilko Verbakel

Netherlands
Frederik Verburg

Germany

Frank Verhaegen

Netherlands

M Vickers

Canada

Salvador Villa

Spain

$\mathrm{N}$ Vivekanandan

India

Erina Vlashi

USA

G Vogin

France

Dirk Vordermark

Germany

Masaru Wakatsuki

Japan

M Wakatsuki

Japan

A Walenkamp

Netherlands

Hui Wang

China

K Wang

USA

Luhua Wang

China

W Weichert

Germany

Robert Weinfurtner

USA

Michael Weller

USA

Stefan Welz

Germany

Thomas G. Wendt

Germany

R Werner

Germany

Moody Wharam

USA

Joachim Widder

Netherlands 
Benedikt Wiestler

Germany

Tilo Wiezorek

Germany

Eric Winquist

Canada

Chris Winter

Switzerland

Lukas Winter

Germany

Florian Wirsdörfer

Germany

Holger Wirtz

Germany

Stefan Wirtz

Germany

Marnix Witte

Netherlands

Matthew Witten

USA

Barbara Witulla

Germany

Suzanne Wolden

USA

Ulrich Wolf

Germany

Jens Wölfelschneider

Germany

Rebecca Wong

Canada
Abraham Wu

USA

Q. Jackie Wu

USA

Hong-Gyun Wu

North Korea

A Wushou

China

Peter Wust

Germany

Ting Xu

USA

S Yahya

UK

Hideomi Yamashita

Japan

Jinzhong Yang

USA

Akihiro Yano

Japan

John Yarnold

UK

Yong Yin

China

Indra Yohannes

Germany

Sua Yoo

USA

K Younge

USA
Angelika Zabel-Du Bois

Germany

Bjorn Zackrisson

Sweden

Nikolaos Zamboglou

Germany

Youssef Zeidan

USA

M Zemanova

Czech Republic

Zhen Zhang

China

Rui Zhang

USA

Chong Zhao

China

Heming Zhen

USA

Thomas Zilli

Switzerland

Horst Zitzelsberger

Germany

Zachary Zumsteg

USA

Brigitte Zurl

Austria

Daniel Zwahlen

Switzerland

Felix Zwicker

Germany 